\title{
EXPERIMENTAL INVESTIGATIONS ON FULL DEPTH BUOYANCY FLOWS IN THE PRESENCE OF SURFACE WAVES
}

\author{
Rosaria Ester Musumeci ${ }^{1}$, Laura Maria Stancanelli ${ }^{2}$, Enrico Foti $^{3}$
}

\begin{abstract}
The propagation of density currents in the presence of waves is experimentally investigated by considering a lockexchange schematization. In particular, we perform experiments considering the classical lock release, where the only driving force is buoyancy, as well as lock-exchange experiments with superimposed periodic surface waves, where the driving forces are both buoyancy and the wave-induced orbital motion. The application of an image processing technique is used for the detection of the main features of the front propagation (i.e depth, height, velocity etc.). All experiments are in full-depth conditions. The propagation of the waves is in intermediate water depth conditions. The presence of surface waves induces an orbital motion along the water column able to modify the dynamics of the density current propagation. Results show that the oscillation of the front advancement, as well as the oscillation of the gravity current depth observed in the presence of waves, are directly correlated with the wave period.
\end{abstract}

Keywords: density current; regular waves; buoyancy

\section{INTRODUCTION}

Gravity currents, called also density currents or buoyancy currents, occur when a fluid flows in a fluid with a different density. Such currents are quite common both in natural and manmade coastal systems (e.g. close to river discharges, to estuaries, or to outflows of industrial plants in the nearshore region).

There is a large body of literature dealing with gravity currents and analyzing the gravity current kinematic characteristics in a broad range of situations. Gravity currents have been studied considering different generating mechanisms: lock-exchange release in full-depth or in partial depth for investigating two-dimensional or three-dimensional gravity current propagation; finite-volume release, mainly "point release", useful for analyzing the axisymmetric propagation of spills. On the other hand, several studies on the influence of ambient fluid flow regime have been presented.

Firstly, the influence of the ambient fluid kinematic characteristics has been investigated in the laboratory by Keulegan (1958), who carried out experiments on gravity currents when an opposite flow is imposed, with the aim to mimic the salt wedge phenomenon in river mouths. Later Britter and Simpson (1978) performed experiments on the gravity currents arrested by a flow, testing the influence of the opposing ambient fluid velocity profile on the gravity current dynamics. In particular, tests were carried out considering both uniform and logarithmic profiles of the opposing ambient fluid. Differences of the ambient fluid dynamics result in the change of the mixing at the front and of the shape of the gravity current itself. Other researches have been focused on different combinations of gravity current generation mechanisms and ambient fluid dynamics, just to name but a few see Arita and Jirka (1987), and Hallworth et al. (1998, 2001).

Robinson et al. (2013) were the first who analyzed experimentally the influence of the orbital motion induced by the presence of regular progressive free-surface water waves on the gravity current propagation. In particular, they considered waves propagating in a flume where a small volume of high density fluid is released to form a gravity current. Analysis on the self-similar phase of the two fronts respectively propagating along the wave direction and in the offshore direction shows the interaction of the gravity current with the wave field. Some preliminary experiments and numerical analysis on the propagation of a lockrelease density current in the presence of regular surface waves have been presented by Musumeci et al. (2017).

The aim of the present work is to investigate the influence on the gravity current dynamics of the ambient fluid regime characterized by the orbital motion induced by intermediate water waves. This is done by reproducing the frequent situation of discharge of fresh or brackish water in the sea, which until now has not been systematically investigated (Robinson et al., 2013). In particular, the present experimental conditions attempt to mimic a continuous outflow of a lighter/fresher fluid in the nearshore region. To this aim, intermediate water conditions superimposed to a steady-state gravity current (slumping phase) are considered. By adopting such a kind of approach, a comparison is possible with the numerous theoretical

\footnotetext{
${ }^{1}$ Department of Civil Engineering and Architecture, University of Catania, Italy

${ }^{2}$ Department of Civil Engineering and Architecture, University of Catania, Italy

${ }^{3}$ Department of Civil Engineering and Architecture, University of Catania, Italy
} 


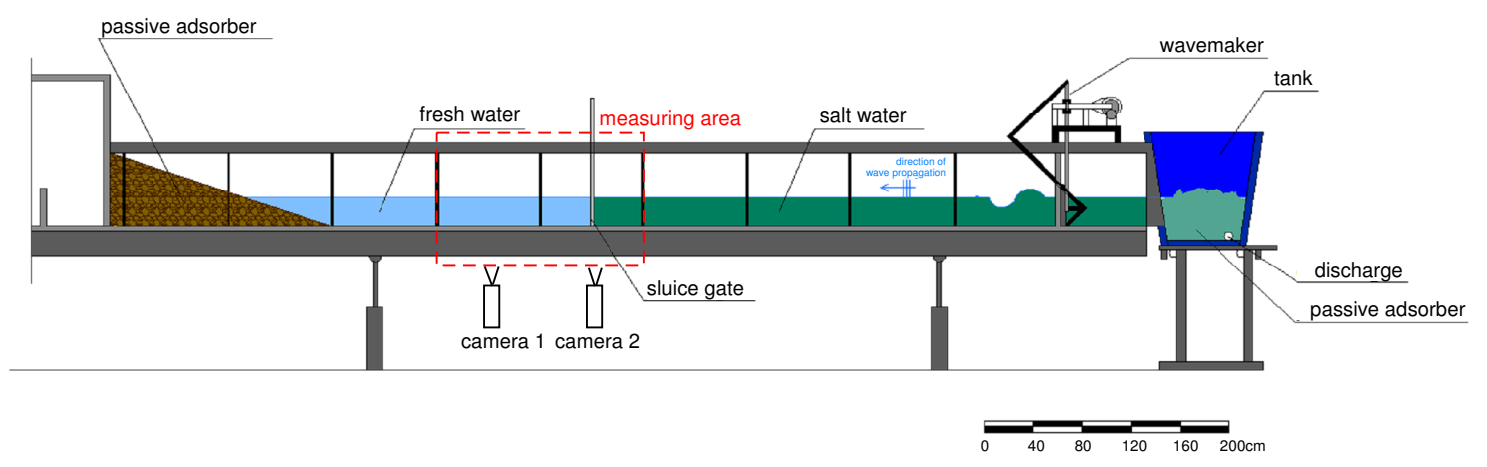

Figure 1: Experimental apparatus for the lock-exchange problems: channel and instruments adopted

and experimental studies on gravity currents produced by the lock-exchange release in the absence of waves. In particular, the application of the classical lock exchange using low aspect ratios, i.e. small values of the water depth compared to the lock length, allows the generation of a constant-speed density front. This permits to clearly highlight the influence of the wave field on the propagation of the gravity current.

The paper is organized as follows. Firstly we describe the experimental apparatus, then the experimental campaign is presented. The experimental evidences are discussed in terms of the gravity current front advancement and height evolution. Finally conclusions and final remarks are given.

\section{EXPERIMENTAL SETUP}

The experiments were carried out at the Hydraulic Laboratory of the University of Catania, in a wave flume which is $9 \mathrm{~m}$ long, $0.5 \mathrm{~m}$ wide and $0.7 \mathrm{~m}$ high (Figure 1). The flume is equipped with a piston-type wavemaker and it has been partitioned at the central section by means of a Perspex sluice gate. At the end of the flume a porous beach allows to minimize wave reflection, as much as possible. Salt water, having density $\rho_{1}$, is present at the wavemaker side of the gate, and fresh water, having density $\rho_{0}<\rho_{1}$, at the onshore side. The sluice gate is located at a distance of $5.3 \mathrm{~m}$ from the wavemaker. The saltier fluid is colored adopting food dye. Laboratory analysis on water samples of the fresh and salted coloured water are carried out to measure the fluid density.

An optical measurement methodology has been used to detect the geometric and kinematic characteristics of the front propagation, such as: depth, velocity and shape. In particular, the area interested by the video recording is about $1.6 \mathrm{~m}$ long in the direction of the current propagation, and it is located at $4.9 \mathrm{~m}$ from the wakemaker (see the red dashed rectangle in Figure 1). The parameters of the wave motion (i.e. wave height, wave period) have been measured by means of four Pepperl and Fuchs acoustic sensors. Velocity profiles have been gathered by means of a Nortek Vectrino Profiler.

\section{EXPERIMENTS}

Twenty four experiments have been carried out to analyze the effects of the wave motion on the propagation of the salt wedge.

The controlling parameters of the experiments are: $h$ still water level within the tank, $\rho_{1}$ salted water density, $\rho_{0}$ fresh water density, $H$ wave height, $T$ wave period, $L$ wave length. In order to analyze the influence of the wave parameters on the gravity current propagation, the experiments have been conducted by considering different reduced gravity $g^{\prime}=g\left(\rho_{1}-\rho_{0}\right) / \rho_{0}$ (range of $g^{\prime}=0.039-0.145 \mathrm{~m} / \mathrm{s}^{2}$ ) and different regular wave conditions (i.e. wave height in the range $1.5-4.3 \mathrm{~cm}$; wave period in the range $0.7 \div 1.3$ $s)$. The above difference of fluid densities assure to reproduce salt wedge propagation in the range of the Boussinesq approximation $\left(\rho_{0} / \rho_{1} \sim 1\right)$. All experiments are in full-depth lock condition, which means that the mean water level is the same at both sides of the gate and that the two different fluids have the same level. The fluid depth $h$ is equal to $20 \mathrm{~cm}$ in all the experiments. Considering the wave parameters described above, it can be noticed that only intermediate water depth conditions have been generated.

Full-depth two-dimensional lock-exchange experiments have been carried out with and without regular waves (Figure 2). In the first case, starting from hydrostatic conditions, the sluice gate is removed and a 

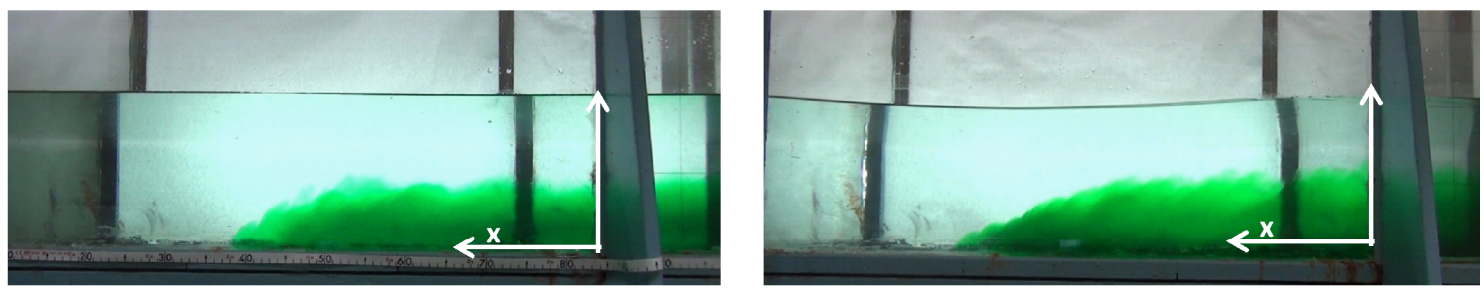

Figure 2: Example of the two type of experiments: a) classical lock release of a brackish wedge, where the only driving force is buoyancy; b) lock release of brackish wedge in the presence of regular waves, where the driving forces are buoyancy and the oscillatory motion.

positive front of denser fluid intrudes in the lower part of the water column within the lighter fluid in the onshore direction while in the upper part a negative front of lighter fluid moves offshore, due to the presence of the density difference $\left(\rho_{1}-\rho_{0}\right)$ (Figure 2a). In the second case, again starting from a hydrostatic condition, the wavemaker generates monochromatic waves which propagate in the onshore direction. As soon as the waves reach the position of the sluice gate, the latter is removed completely and a gravity current develops. In this case, the propagation of the saltier front in the onshore direction occurs in the presence of a wave motion (Figure $2 \mathrm{~b}$ ). Since the sluice gate is a perfectly reflecting vertical wall, in order to minimize spurious effects due to wave reflection, the removal of the gate is performed just before the impact of the first wave.

\section{RESULTS}

A preliminary analysis on the reflection phenomena in the tank, using data coming from the acoustic sensors, has been performed by applying the well-know Mansard and Funke (1980) three-probe method and the recently proposed four-probe method of Faraci et al. (2014). Both methods show similar results and a reflection coefficient which in all cases is lower than 0.13 .

The experimental evidences about the dynamics of the gravity current propagation have been acquired thanks to the the application of an image processing methodology. Starting from the video records of the experiments acquired at a resolution of 50fps, firstly the extraction of all frames is performed (see an example in Figure 3a), then an automated image processing is carried out. In particular, the images are cropped to select the area of interested by the dynamics of the gravity current propagation and adjustments of image distortion are also applied (see Figure 3b). Later each image is transformed in black and white, to allow an easier identification of the gravity current shape and of the front position (Figure 3c). The accurate detection of the head and of the body of the propagating gravity current is relevant for identifying the front advancement and the part of the water column occupied by the density current.

Results on the front advancement in the case of the classical lock exchange problem confirm, as expected, that an increment of the reduced gravity is responsible of an increase of the current propagation velocity. The observed linear time evolution of the front advancement (see Figure 4a) indicates that we are observing a gravity current during the constant-velocity phase, namely during the slumping phase (Shin et al., 2004). The mean front velocity can be determined as the slope of the linear best fit of the experimental data. As expected an increase of the difference of fluid density correspond to an increase of the propagating velocity, ranging from about $4 \mathrm{~cm} / \mathrm{s}$ to about $7 \mathrm{~cm} / \mathrm{s}$ in the correspondence of changes of $g^{\prime}$ from $0.04 \mathrm{~m} / \mathrm{s}^{2}$ to $0.14 \mathrm{~m} / \mathrm{s}^{2}$. The experimental data in Figure 4a fit well the theoretical prediction of Huppert and Simpson (1980), derived for similar conditions.

In the presence of regular surface waves the dynamics of the front shows a pulsating behavior, induced by the oscillating motion, with the period of the pulsations being equal to the wave period. The time evolution of the front propagation is presented in Figure $4 \mathrm{~b}, \mathrm{c}, \mathrm{d}$ and it is compared with the theoretical advancement predicted by Huppert and Simpson (1980) in the absence of waves. As opposite to the case of initially quiescent ambient fluid, it appears that when surface waves are present the data deviate significantly from the theory. In particular, in the presence of the wave motion, the experimental results indicate that an increase of the wave period (or a decrease of the wave steepness) leads to a decrease of the mean velocity of gravity current propagation. For example, in Figure $4 \mathrm{~d}$ the maximum decrease of the average front velocity is of about $20 \%$.

The superposition of the surface wave motion induces also the oscillation of the interface between the 
a)

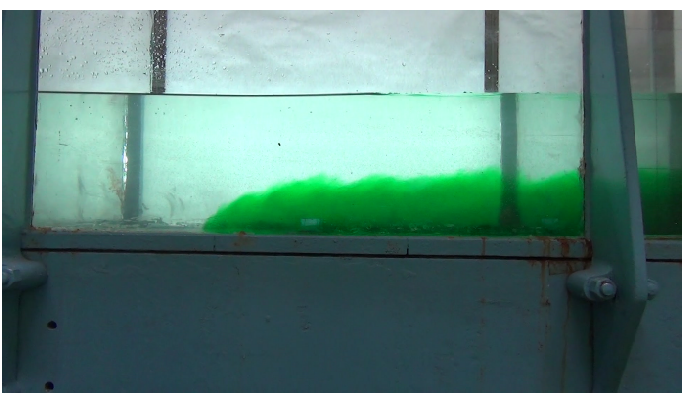

c)
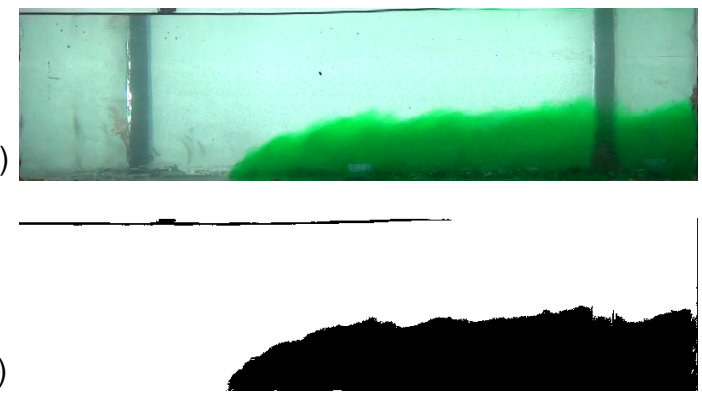

Figure 3: Salt wedge front advancement: a) image acquired in laboratory; b) post processing crop and distortion adjustment; c) black and white transformation useful for the front detection.
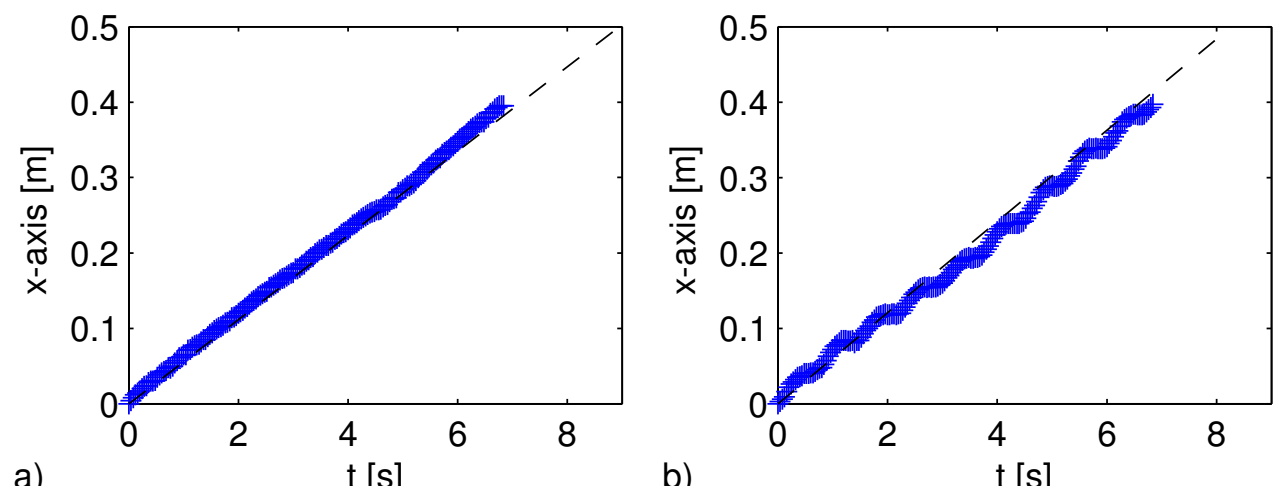

b)
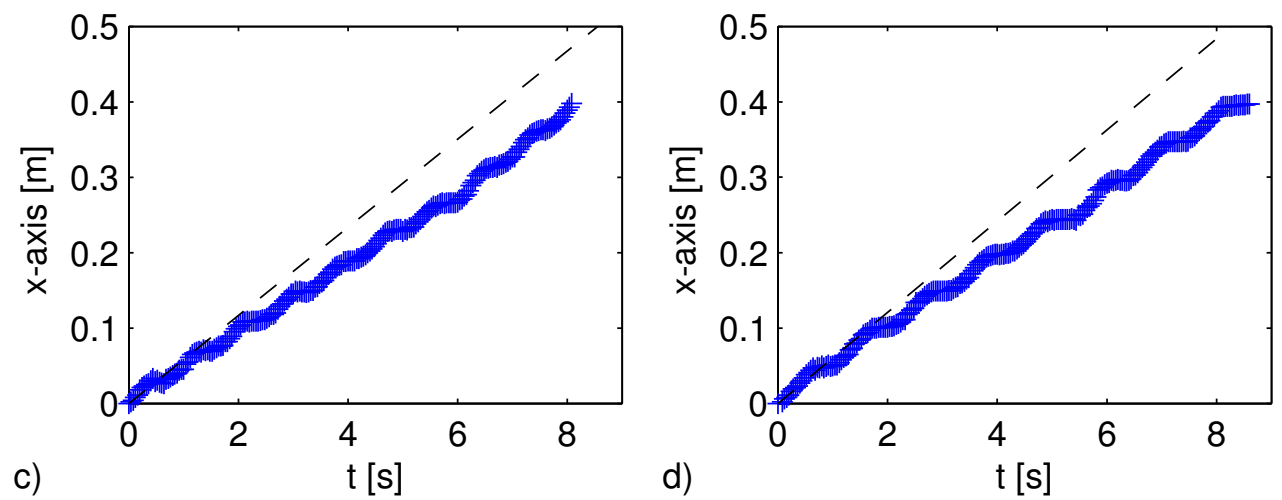

Figure 4: Time evolution of salt wedge front advancement: a) in initially quiescent ambient fluid characterized by an initial reduced gravity $g^{\prime}$ of $0.08 \mathrm{~m} / \mathrm{s}^{2} ; \mathbf{b}$ ) in the presence of regular waves $H=4.22 \mathrm{~cm} T=0.71$ $s$, characterized by reduced gravity $g^{\prime}$ of $0.09 \mathrm{~m} / \mathrm{s}^{2}$; c) in the presence of regular waves $H=3.00 \mathrm{~cm} T=0.84$ $s$, characterized by reduced gravity $g^{\prime}$ of $0.09 \mathrm{~m} / \mathrm{s}^{2} ; \mathbf{d}$ ) in the presence of regular waves $H=2.54 \mathrm{~cm} T=1.02$ $s$ and characterized by reduced gravity $g^{\prime}$ of $0.09 \mathrm{~m} / \mathrm{s}^{2}$. Dashed lines indicate the theoretical advancement profile from Huppert and Simpson (1980) determined for gravity current of equal reduced gravity and propagating in quiescent ambient fluid during constant velocity phase. 

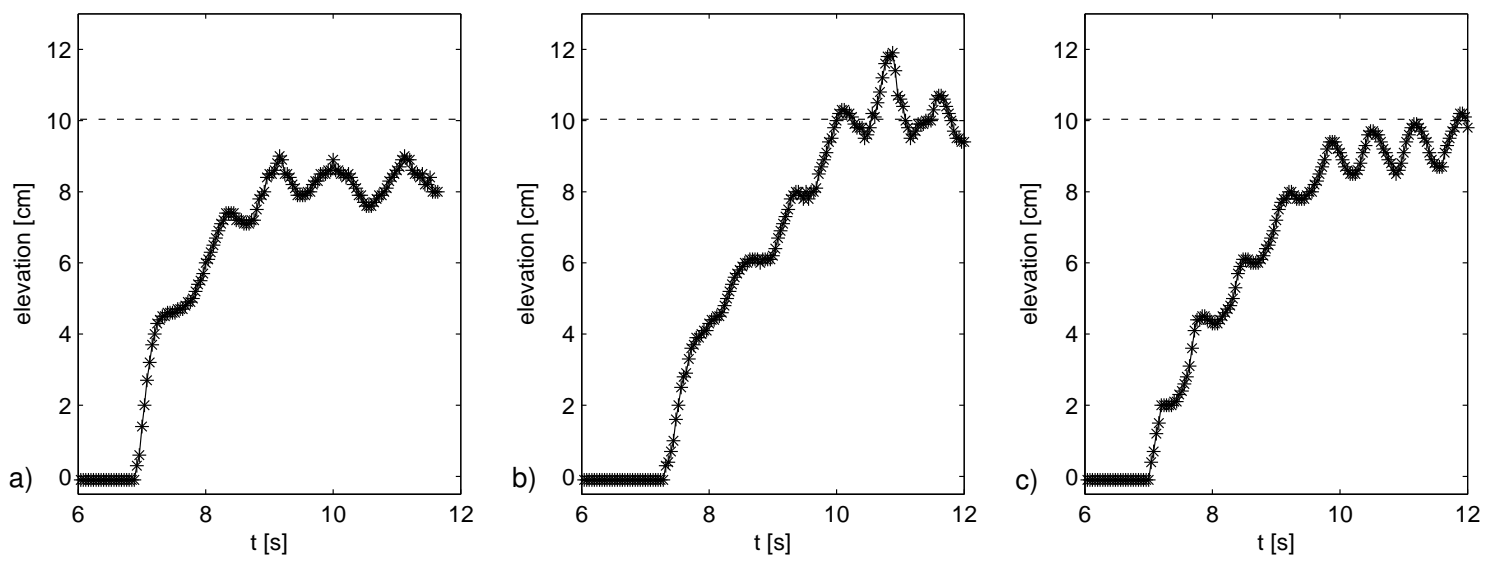

Figure 5: Time evolution of salt wedge height acquired in the presence of regular waves at a fixed section (located $6.1 \mathrm{~m}$ offshore from the wavemaker). For experiments in presence of waves characterized by: a) $\left.\left.g^{\prime}=\mathbf{0 . 0 1 3} \mathrm{m} / \mathrm{s}^{2}, H=\mathbf{2 . 4 5} \mathrm{cm} T=\mathbf{1 . 0 2} \mathrm{s} ; \mathbf{b}\right) g^{\prime}=\mathbf{0 . 0 1 3} \mathrm{m} / \mathrm{s}^{2}, H=\mathbf{2 . 8 6} \mathrm{cm} T=\mathbf{0 . 8 4} \mathrm{s} ; \mathbf{c}\right) g^{\prime}=\mathbf{0 . 0 1 3} \mathrm{m} / \mathrm{s}^{2}, H=3.93 \mathrm{~cm}$ $T=0.71 s$. Dash lines indicated the reference of half lock height $(H / 2)$.

ambient fluid and the gravity currents is observed. Indeed, as shown in Figure 5, the time evolution of salt wedge height acquired in the presence of regular waves at a fixed section, located $6.1 \mathrm{~m}$ offshore of the wavemaker, is influenced by the orbital motion along the water column. The dimensions of such oscillations $(\mathrm{O}(<1 \mathrm{~cm}))$ are a function of the superimposed wave regime and interact with the shear layer development at the interface between the two flows. In particular, the frequency of the oscillation appears again to be related at leading order to the wave period.

\section{CONCLUSIONS}

An experimental investigation on gravity currents dynamics in the presence and in the absence of regular surface waves has been carried out. By means of image processing technique, it was possible to acquire data about advancement of the front, shape of the current, as well as the height of the current at fixed section. The experiments in the absence of waves provide information about the mean velocity advancement of the gravity current for the selected range of the density differences between the two fluids. In this case the constant velocity of the front advancement suggests that we were observing gravity currents in the slumping phase. Experiments in the presence of wave reveals that the ambient fluid dynamics influence the dynamics of the generated gravity currents to a large extent. Comparisons with the theory of Huppert and Simpson (1980) for predicting the front advancement in the case of initially quiescent ambient fluid and in the slumping phase shows that the wave motion caused a decrease of the average velocity of the heavier front. Moreover the advancement of the front in the case of waves was no linear anymore, but it became pulsating with a characteristic frequency that is similar to the one of the wave period. The gravity current elevation acquired at a fixed section for different experimental conditions showed that also the interface of the gravity current is influenced by a regular oscillation, which seams to be related to the wave period and to the amplitude of the orbital trajectories. Such an oscillation is not present in the case of initially quiescent ambient fluid.

In conclusion, the present experimental results have shown that the effect of orbital motion may be significant on the propagation of gravity currents and affect also important coastal processes, such as the transport of sediments, nutrients and contaminants. Indeed, the highest values of the velocity due to the pulsating behavior of the front could be responsible of triggering or increasing sediment transport over movable coastal beds. On the other hand, the wave-induced oscillations of the internal interface could accelerate mixing processes along the water column.

\section{ACKNOWLEDGEMENTS}

This work has been partly funded by the Italian Ministry of Education, Universities and Research MIUR through the Research projects of significant national interest PRIN 2010 - 2011 project name HYDROCAR (cod. 20104J2Y8M003) and by the PRIN 2012 project Project "Hydro-morphodynamics mod- 
elling of coastal processes for engineering purposes" (cod. 2012BY TPR5), by the EU funded project HYDRALAB PLUS (proposal number 64110), and by the PO FESR SICILIA 2007 - 2013, Axes IV, Project MedNETNA.

\section{References}

M. Arita and G. H. Jirka. Two-layer model of saline wedge. ii: Prediction of mean properties. Journal of Hydraulic Engineering, 113(10):1249-1263, 1987.

R. Britter and J. Simpson. Experiments on the dynamics of a gravity current head. Journal of Fluid Mechanics, 88(02):223-240, 1978.

C. Faraci, P. Scandura, and E. Foti. Reflection of sea waves by combined caissons. Journal of Waterway, Port, Coastal, and Ocean Engineering, 141(2):04014036, 2014.

M. A. Hallworth, A. J. Hogg, and H. E. Huppert. Effects of external flow on compositional and particle gravity currents. Journal of Fluid Mechanics, 359(1):109-142, 1998.

M. A. Hallworth, H. E. Huppert, and M. Ungarish. Axisymmetric gravity currents in a rotating system: experimental and numerical investigations. Journal of Fluid Mechanics, 447:1-29, 2001.

H. E. Huppert and J. E. Simpson. The slumping of gravity currents. Journal of Fluid Mechanics, 99(04): 785-799, 1980.

G. H. Keulegan. Twelfth progress report on model laws for density currents, volume 5831. US Dept. of Commerce, National Bureau of Standards, 1958.

E. P. Mansard and E. Funke. The measurement of incident and reflected spectra using a least squares method. Coastal Engineering Proceedings, 1(17), 1980.

R. E. Musumeci, A. Viviano, and E. Foti. Influence of regular surface waves on the propagation of gravity currents: experimental and numerical modelling. Accepted for pubblication in Journal of Hydraulic Engineering, 2017.

T. Robinson, I. Eames, and R. Simons. Dense gravity currents moving beneath progressive free-surface water waves. Journal of Fluid Mechanics, 725:588-610, 2013.

J. Shin, S. Dalziel, and P. Linden. Gravity currents produced by lock exchange. Journal of Fluid Mechanics, 521:1-34, 2004. 